
\title{
25 Research Square \\ Ti(IV)-exchanged nano-ZIF-8 and nano-ZIF-67 for Enhanced Photocatalytic Oxidation of Hydroquinone
}

\section{Lehlohonolo E. Mphuthi}

University of the Free State Faculty of Natural and Agricultural Sciences

Mametsi R. Maseme

University of the Free State Faculty of Natural and Agricultural Sciences

Ernie H.G. Langner ( $\nabla$ langneeh@ufs.ac.za )

University of the Free State Faculty of Natural and Agricultural Sciences https://orcid.org/0000-00024667-3396

\section{Research Article}

Keywords: nano-ZIF-8, nano-ZIF-67, Ti(IV)-exchange, Photocatalysis, Oxidation, Hydroquinone

Posted Date: January 5th, 2022

DOI: https://doi.org/10.21203/rs.3.rs-1215825/v1

License: (c) (1) This work is licensed under a Creative Commons Attribution 4.0 International License.

Read Full License 


\section{Abstract}

The metal centres of nano-ZIF-8(Zn) and nano-ZIF-67(Co) were partially exchanged with titanium centres to form bimetallic nZIF-8(Zn/Ti) $\left(52 \% \mathrm{Ti}^{4+}\right)$ and nZIF-67(Co/Ti) $\left(38 \% \mathrm{Ti}^{4+}\right)$ respectively, for enhanced photocatalytic performance. A morphological and structural analysis by SEM, EDS-Mapping and PXRD showed that the particle size, distribution, and the structural integrity of the Sodalite frameworks of the parent ZIFs were retained during the exchange process to form the new bimetallic Ti-ZIFs. FTIR confirmed that no additional chemical bonds were formed during the process. XPS binding energies confirmed the preservation of the $\mathrm{Zn}(\mathrm{II}), \mathrm{Co}(\mathrm{II})$ and $\mathrm{Ti}(\mathrm{IV})$ oxidation states, as well as the Ti-content, consistent with ICPOES and EDS measurements. The Ti-exchanged ZIFs showed higher activity during the photocatalytic oxidation of hydroquinone in comparison with their parent ZIFs. Their kinetic rates were nearly five times faster than those of the parent ZIFs, with the first-order rate constants $\mathrm{k}=0.189 \mathrm{~min}^{-1}$ for nZIF-8( $\left.\mathrm{Zn} / \mathrm{Ti}\right)$ and $\mathrm{k}=0.139 \mathrm{~min}^{-1}$ for $\mathrm{nZIF-67(Co/Ti)}$. These catalysts are efficient, stable, and reusable for three photocatalytic cycles without a significant loss of catalytic activity.

\section{Introduction}

Metal-organic frameworks (MOFs) are interesting family of materials that have attracted considerable attention because of their distinctive and highly desired properties such as high surface area, large and tuneable pore sizes. They can be synthesized using a variety of metal ions and organic linkers $[1,2]$. The number of novel MOFs has recently expanded substantially, with metal components spanning the periodic table from s-block elements through transition metals to rare-earth metals [3], those with transition metal clusters being the most common [4].

Zeolitic imidazolate frameworks (ZIFs), a subfamily of MOFs, made up of metal ions linked by imidazolate derivatives, are effective for a range of applications, including catalysis [5]. Despite their high catalytic activity, monometallic ZIFs are rarely employed in photocatalysis due to their limited photocatalytic activity in most reactions [6]. Their photocatalytic activity can be enhanced by introducing an additional photocatalytic active transition metal to the framework, resulting in a bimetallic catalytic system with hetero active sites [7]. Our group has effectively investigated the effect of a two metal system for increased photocatalytic degradation of RBBR textile dye by partially exchanging $\mathrm{Zn}^{2+}$ and $\mathrm{Co}^{2+}$ ions in ZIF-8 and ZIF-67 with a redox active $\mathrm{Fe}^{2+}$ centre, respectively [8]. This paper will investigate the effect of incorporating Ti centres into ZIF materials.

Herein, we focus on modifying nano-ZIF-8(Zn) and nanoZIF-67(Co) via postsynthetic metal $\left(\mathrm{Ti}^{4+}\right)$ exchange to produce novel bimetallic Ti-ZIF frameworks that are photocatalytically more active than their parent ZIFs. Directly synthesized Ti-MOFs are usually structurally unpredictable, making cation exchange a viable approach for making Ti-MOFs while largely retaining the porosity and/or topological structures of the parent MOFs. In the first examples of Ti-exchange, UiO- 66 was exchanged with $\mathrm{Ti}^{4+}$. It was 
confirmed that the MOF particles contain $\mathrm{Ti}^{4+}$ (roughly $38 \mathrm{wt} \%$ ) during post-synthetic cation exchange. Furthermore, the crystallinity of Ti-exchanged UiO-66( $\mathrm{Zr} / \mathrm{Ti})$ was intact [9].

Tetravalent titanium $\left(\mathrm{Ti}^{4+}\right)$ appears to be favourable for the synthesis of MOFs with high redox activity, chemical stability, and exceptional photocatalytic properties [10]. Furthermore, MOFs based on titaniumoxo clusters are fascinating materials that, due to their outstanding durability at high temperatures, high humidity, and harsh chemical conditions, could replace $\mathrm{TiO}_{2}$ in a variety of industrial applications [11, 21]. The synthesis of Ti-based MOFs, however, is difficult due to the extremely reactive nature of titanium precursors [13]. Controlling the reactivity of Ti precursors is crucial in the production of Ti-MOFs, which involves careful solution combination, temperature, and time selection [14].

Ti-MOFs have numerous distinguishing features that set them apart from other MOFs, such as promising photoresponsivity properties [14]. Their low toxicity makes them suitable for a wide range of photocatalytic applications and commercial uses $[15,16]$. Due to their challenging synthesis and lack of control over their chemistry and crystallization, Ti-based ZIFs with $\mathrm{Ti}^{4+}$ centres have remained elusive for years $[17,18]$. To the best of our knowledge, none of the current solvent-based ZIF synthesis methods was adapted for the synthesis of Ti-containing ZIFs.

\section{Experimental}

\subsection{Materials and equipment}

Starting materials and solvents were purchased from Sigma-Aldrich and Merck with high purity and used without further puri $\square$ cation, except were stated otherwise. A Bruker Tensor 27 infrared spectrophotometer, equipped with a Pike Miracle single diamond ATR crystal, was used to measure all infrared spectra. A JSM-7800F Extreme-resolution Analytical Field Emission SEM, fitted with an Oxford XMax $\left(80 \mathrm{~mm}^{2}\right)$ EDS, was used to examine and analyze SEM specimens. Micromeritics' ASAP 2020 Surface Area and Porosity Analyzer was used to measure porosity and surface area up to $1 \mathrm{~atm}$, and the data was analyzed using ASAP 2020 V2.0 and Micro-active software for physisorption. Prior to porosity measurements, the nanoZIF materials were activated at $150^{\circ} \mathrm{C}$ for 16 hours, and a standard amount of $\sim 40 \mathrm{mg}$ was used for each analysis, with warm and cold free space calculated separately. PXRD patterns were collected at room temperature on a Bruker D2 Phaser powder X-ray diffractometer with a flat plate sample holder and $\mathrm{Cu}$ radiation $(\lambda=1.54 \AA$ ) [17]. TGA was carried out on a Mettler-Toledo TGA/SDTA851 in a nitrogen atmosphere at a heating rate of $10^{\circ} \mathrm{C} \cdot \mathrm{min}^{-1}$ from $30^{\circ} \mathrm{C}$ to $800^{\circ} \mathrm{C}$. With a monochromatic Al K X-ray source, XPS was recorded on a PHI 5000 Versaprobe system. The XPS data was analyzed with Multipak version 9.7c software, and Gaussian-Lorentz fits were used (the Gaussian/Lorentz ratios were always $>95 \%$ ). UV-vis measurements were done using a Shimadzu CPS-240A UV-vis spectrophotometer [8, 23, 24].

\subsection{Synthesis of ZIF nanoparticles in methanol}


Nano-ZIF-8 and nano-ZIF-67 crystallites were synthesized in methanol by a rapid isothermal bench top reaction and activated thereafter according to a recently published method (Mphuthi et al) ${ }^{8}$ (Scheme 1). Yield: $0.827 \mathrm{~g}, 63 \%$ (nZIF-8) and $1.572 \mathrm{~g}, 61 \%$ (nZIF-67). $\mathrm{v}_{\max } / \mathrm{cm}^{-1}$ (Fig. 1): v(C-H) = 2930; 3138, v(C-N) = $1151 ; 1039, v(C=N)=1589$. PXRD (Fig. 2) $[19,20,21]$.

Scheme 1. Isothermal synthesis of nano-ZIF-8 and nano-ZIF-67 from metal(Zn/Co) nitrate hexahydrate and 2-methylimidazole.

\subsection{Metal-ion exchange between ZIFs and Ti(IV) isopropoxide (Inert conditions)}

Scheme 2. Ti(IV)-Exchange of nano-ZIF-8(Zn) and nano-ZIF-67(Co) crystallites.

\section{Methanol Drying procedure}

Dry magnesium turnings $(2.5 \mathrm{~g})$ and iodine $(0.25 \mathrm{~g})$ were added to $50 \mathrm{~cm}^{3}$ methanol. After the iodine disappeared and magnesium converted to methanolate due to heating, $350 \mathrm{~cm}^{3}$ methanol was added and refluxed for an hour. Dry methanol was distilled under $\mathrm{N}_{2}$ and collected.

\section{Postsynthetic exchange}

Since the Ti(IV) isopropoxide precursor is extremely reactive, $\mathrm{ZIF-8}(\mathrm{Zn} / \mathrm{Ti})$ and $\mathrm{ZIF-67(Co/Ti)}$ nanoparticles were synthesised under inert conditions by a modified procedure, described by Fei et al. [22]. A two-neck flask was dried and fitted with a condenser and rubber septum and purged with nitrogen gas for 30 minutes before the reagents were added. In a glove box, under anhydrous conditions, titanium(IV) isopropoxide $\left(1.8 \mathrm{~cm}^{3}, 6 \mathrm{mmol}\right)$ was dissolved in $50 \mathrm{~cm}^{3}$ dry methanol using an ultrasonic bath. To this solution, nZIF (ca. $0.45 \mathrm{~g}, 2$ mmol equiv. of Zn for nZIF-8: ca. $0.44 \mathrm{~g}, 2$ mmol equiv. of Co for nZIF-67) was added. The reagent mixture was transferred to a two-neck flask and heated for $24 \mathrm{hrs}$ in a prepared oil bath at $60^{\circ} \mathrm{C}$, while stirring at $150 \mathrm{rpm}$ under $\mathrm{N}_{2}$. The solid products were collected by centrifugation $\left(8500 \mathrm{rpm}, 10 \mathrm{~min}, 15^{\circ} \mathrm{C}\right.$ ), after cooling to room temperature. Methanol was used to wash the precipitate until the supernatant was colourless. The solids were soaked in methanol for three days, and the solution was changed every $24 \mathrm{hrs}$ with fresh methanol. The solid products were centrifuged until the supernatant was colourless, and dried at $60^{\circ} \mathrm{C}$, before activation at $150^{\circ} \mathrm{C}$ overnight under vacuum, to obtain nZIF-8(Zn/Ti) and nZIF-67(Co/Ti).

\subsection{Photocatalytic Application (Oxidation of hydroquinone $\left(\mathrm{H}_{2} \mathrm{Q}\right)$ )}

The photocatalytic activity of Ti-exchanged nZIF-8(Zn/Ti) and nZIF-67(Co/Ti), compared to their parent nZIF-8 ( $\mathrm{Zn})$ and nZIF-67(Co), was investigated by the oxidation of hydroquinone $\left(\mathrm{H}_{2} \mathrm{Q}\right)$ under simulated sunlight irradiation, using a $350 \mathrm{~W}$ Metal Halide Lamp (placed at $10 \mathrm{~cm}$ distance from the reactor), under $\mathrm{N}_{2}$ at room temperature. Both dark and photocatalytic reactions were carried out to differentiate between 
the effect of adsorption and the photocatalytic oxidation by the synthesized catalysts. To a suspension of hydroquinone $(120 \mathrm{mg}, 1.1 \mathrm{mmol})$, catalyst (ZIF materials, $50 \mathrm{mg}$ ), and Acetonitrile $\left(\mathrm{CH}_{3} \mathrm{CN}, 10 \mathrm{~cm}^{3}\right)$, in a $100 \mathrm{~cm}^{3}$ two-neck round bottom flask purged with $\mathrm{N}_{2}$ and stirred for 5 min to reach homogeneity, a solution of $30 \%$ aq. $\mathrm{H}_{2} \mathrm{O}_{2}\left(0.5 \mathrm{~cm}^{3}\right)$ was added dropwise under stirring. The samples were collected at 3 min time intervals and diluted with Acetonitrile for UV-Vis spectrophotometry analyses. Blank experiments were performed, firstly in the absence of catalyst and secondly in the absence of $\mathrm{H}_{2} \mathrm{O}_{2}$ to verify the stability of $\mathrm{H}_{2} \mathrm{Q}$ and to confirm that no significant sorption of the substrate occurred for either the experimental equipment or the catalyst. The isolated benzoquinone product was identified with FTIR [23].

\section{Results And Discussion}

A rapid isothermal bench top reaction was used to synthesize nano-ZIF-8 and nano-ZIF-67 crystallites in methanol. The process starts with the deprotonation of 2-methylimidazole (2-MeIM), which subsequently coordinates tetrahedrally to the $\mathrm{Zn}^{2+}$ or $\mathrm{Co}^{2+}$ ions to form nZIF-8(Zn) or nZIF-67(Co) crystallites, respectively. Since ZIF-67 particles are usually larger in size, PVP-10 was used to inhibit particles growth, leading to the formation of nanoparticles. Triethylamine (TEA) was used to accelerate the deprotonation of 2-Melm, leading to faster nucleation and thus promote the formation of nano-sized ZIFs. During metal exchange, $\mathrm{Ti}(\mathrm{IV})$-isopropoxide was employed as a source of $\mathrm{Ti}^{4+}$ to partially substitute $\mathrm{Zn}^{2+}(\mathrm{ZIF}-8)$ and $\mathrm{Co}^{2+}$ (ZIF-67), coordinating tetrahedrally to 2-MelM, to produce bimetallic nZIF-8(Zn/Ti) and nZIF$67(\mathrm{Co} / \mathrm{Ti})$ crystallites respectively. The presence of Ti(IV) in nZIF-8 and nZIF-67 was confirmed by Inductively Coupled Plasma-Optical Emission Spectroscopy (ICP-OES), X-ray Photoelectron Spectroscopy (XPS) and Scanning electron microscopy-energy-dispersed X-ray spectroscopy (SEM-EDX).

The obtained Ti-exchanged ZIF materials were confirmed to be free of unreacted Ti(IV) isopropoxide by the FTIR. As shown in Figure 1, all Titanium exchanged ZIFs, nano-ZIF-8(Zn/Ti) and nano-ZIF-67(Co/Ti), exhibit FTIR spectra similar to the pristine ZIFs. The characteristic bands for Ti(IV) isopropoxide are highlighted; (i) Asymmetric and symmetric stretching of the isopropoxide - $\mathrm{CH}$ group at 2900 and 2800 $\mathrm{cm}^{-1}$ (ii) $1420 \mathrm{~cm}^{-1}$ (C-O stretching) and (iii) $928 \mathrm{~cm}^{-1}$ (C-O bending). The broad band in the range of $500-700 \mathrm{~cm}^{-1}$ could be attributed to vibrations of the Ti-O and TiO-Ti bonds. The absence of these bands in the spectra of ZIFs after metal exchange reaction strongly indicates the absence of unreacted $\mathrm{Ti}(\mathrm{IV})$ isopropoxide in the Ti-exchanged products. Furthermore, no new bands are identified when compared to the parent ZIFs, indicating that no additional bonds were formed during the exchange [8].

\section{Figure 1}

FTIR spectra of (a) nZIF-8(Zn) with nZIF-8(Zn/Ti) and (b) nZIF-67(Co) with nZIF-67(Co/Ti).

The similar PXRD patterns of pristine nZIF-8 and nZIF-67 nanoparticles and their Ti-exchanged counterparts, confirmed the preservation of structural integrity of nZIF-8 and nZIF-67 after metal 
exchange with Ti(IV) (Fig. 2). The crystallinity and Sodalite (SOD) topologies of all ZIFs were confirmed by their well-defined PXRD patterns, which show comparable patterns and are in good accord with the simulated pattern [26-30]. These findings indicate that nano-ZIF-8 and nano-ZIF-67 are relatively stable, with their crystal structures being preserved to a great degree throughout the Ti(IV) exchange [31].

\section{Figure 2}

PXRD patterns of (a) nZIF-8(Zn) with nZIF-8(Zn/Ti) and (b) nZIF-67(Co) with nZIF-67(Co/Ti).

SEM-EDX was used to study the morphology of pristine and Ti-exchanged nZIF-8 and nZIF-67, as well as the dispersity of $\mathrm{Ti}(\mathrm{IV})$ in the exchanged products (Fig. 3). The particle size and morphology of pristine (left) and Ti-exchanged (right) ZIFs, show very little change. In agreement with the literature, the SEM images of nZIF-8 crystals (Fig. 3a) exhibit smooth hexagonal, nano-sized structures [28, 32-34]. The average particle diameter of nZIF-8 is $48 \mathrm{~nm}$ and $62 \mathrm{~nm}$ for the Ti-exchanged nZIF-8(Zn/Ti). In Figure 3(b and e), SEM images of Ti-exchanged ZIFs exhibit nano-sized crystallites similar to the parent ZIFs. The nZIF-67 crystals are usually larger than nZIF-8 crystals, averaging $109 \mathrm{~nm}$ for pristine nZIF-67 and 126 $\mathrm{nm}$ for the Ti-exchanged nZIF-67(Co/Ti) (Fig. 3d). The nano-sized, rhombic dodecahedral nZIF-67 crystallites were obtained by using PVP-10, shaped in suspended micelles via their hydrophilichydrophobic interactions [29, 30,34]. SEM-EDX mapping of Ti-exchanged ZIFs clearly shows a consistent and even distribution of C, N, Zn, Co and Ti (Fig. 3c and 3f), which is quite similar to the EDX-mapping of parent ZIFs (Supporting Information, Fig. S1). All elements are equally spread on the surface of the materials, as seen on the mapping, indicating that metal exchange occurred uniformly on the crystal surface [35].

\section{Figure 3}

SEM images of (a) ZIF-8(Zn), (b) nZIF-8(Zn/Ti), (d) nZIF-67(Co), (e) nZIF-67(Co/Ti) and EDX-mapping of Ti-exchanged nZIF-8(Zn/Ti) (C1-C4) and nZIF-67(Co/Ti) (F1-F4).

All ZIF materials were subjected to high resolution X-ray Photoelectron Spectroscopy (XPS) measurements to determine the elemental composition, oxidation states of metals and atomic ratios of elements present within the samples. The comparative XPS data of pristine nZIF-8(Zn) and nZIF-67(Co) with the Ti-exchanged nZIF-8(Zn/Ti) and nZIF-67(Co/Ti) (Table 1) shows the binding energies of the $\mathrm{N} 1 \mathrm{~s}$, Zn $2 p$, Co $2 p$ and Ti $2 p$ photoelectron lines.

The XPS binding energy scale often requires charge referencing, due to sample charging during the measurement, often shifting core-level peaks towards higher binding energy values. Also, air-exposed samples always contain carbon and oxygen surface contaminants which need to be accounted for. The $\mathrm{C}$ $1 \mathrm{~s}$ binding energy in particular depends on the types of hydrocarbons that are adsorbed on the surface of the sample causing a charge shift that may lead to inaccurate peak allocation [36,37]. To correct this, the maximum binding energy of carbon's C 1 s photoelectron line, at $284.8 \mathrm{eV}$, was used as a charge reference for all the binding energies $[23,37,38]$. 
All ZIF materials show a symmetrical N 1s photoelectron between 398.6-398.9 eV, assigned to the collective nitrogen species components in the imidazole ring namely $\mathrm{N}=\mathrm{C}, \mathrm{N}-\mathrm{C}, \mathrm{N}-\mathrm{M}^{\mathrm{n}+}$ groups where $\mathrm{n}=$ 2 or 4 (Fig. 4a) [40]. The zinc-containing nZIF-8(Zn) and nZIF-8(Zn/Ti) compounds showed the Zn-2 $\mathrm{p}_{3 / 2}$ photoelectron line between 1021.4-1021.9 eV with a spin orbit splitting of ca. $23.1 \mathrm{eV}$ between the $\mathrm{Zn}$ $2 p_{3 / 2}$ and $Z n-2 p_{1 / 2}$ photoelectron lines (Fig. $4 \mathbf{b}$ ). The observed binding energies confirms the presence of $\mathrm{Zn}^{2+}$ and are compatible with $\mathrm{Zn}^{2+}$ binding energy observed for other reported ZIF-8 structures [25, 38, 39]. For cobalt containing nZIF-67(Co) and nZIF-67(Co/Ti) compounds, XPS revealed the $\mathrm{Co}^{-2} \mathrm{p}_{3 / 2}$ and the Co- $2 \mathrm{p}_{1 / 2}$ photoelectron line between 781.1-781.4 eV and 786.4-786.7 eV, respectively (Fig. 4d). The Co- $2 p_{3 / 2}$ binding energy shows that cobalt is in the redox state +2 , and correlates well with reported Co$2 \mathrm{p}_{3 / 2}$ binding energy values for compounds that contain $\mathrm{Co}-\mathrm{N}$ bonds such as $\mathrm{Co}\left(\mathrm{NH}_{3}\right)_{6} \mathrm{Cl}_{3}$ found at $781.1 \mathrm{eV}[37,42]$. Satellite features were also observed at ca. $4.9 \mathrm{eV}$ higher than the main Co-2 $\mathrm{p}_{3 / 2}$ and the Co- $2 \mathrm{p}_{1 / 2}$ photoelectron lines, due to the shake-up effect caused by the reduction of the emitted photoelectron kinetic energy. The intensity of the satellite to the main photoelectron lines were ca. $30 \%$ with the intensity increasing after the metal exchange reaction occurred. For Titanium containing nZIF$8\left(\mathrm{Zn} / \mathrm{Ti}\right.$ ) and nZIF-67(Co/Ti) (Fig. 4c), the XPS showed the Ti-2 $\mathrm{p}_{3 / 2}$ and Ti-2 $\mathrm{p}_{1 / 2}$ photoelectron line set at ca. 459.5 and $466.9 \mathrm{eV}$, which is clearly defined by a characteristic peak of $\mathrm{Ti}(\mathrm{IV})[43,44]$. The presence of these photoelectron lines confirmed successful metal exchange, and the binding energy value implies that $\mathrm{Ti}$ is in an oxidation state of (IV). From the \% atomic ratios, the success of the metal exchange reaction was found to be $52 \%$ for $\mathrm{nZIF-} 8(\mathrm{Zn} / \mathrm{Ti})$ and $38 \%$ for $\mathrm{nZIF-67}(\mathrm{Co} / \mathrm{Ti})$, respectively. No satellite features were observed for titanium.

\section{Figure 4}

The (a) N 1s, (b) Zn 2p, (c) Ti 2p and (d) Co 2p XPS regions of ZIF-8, ZIF-67 and their Ti-exchanged derivatives. 
Table 1

XPS data of nZIF-8, nZIF-67, and their Ti-exchanged derivatives.

\begin{tabular}{|llllllllll|}
\hline & N & Zn & \multicolumn{3}{c}{ Co(main) } & Co(sat) & \multicolumn{2}{l|}{ Ti(main) } \\
\hline & $1 \mathrm{~s}$ & $2 \mathrm{p}_{3 / 2}$ & $2 \mathrm{p}_{1 / 2}$ & $2 \mathrm{p}_{3 / 2}$ & $2 \mathrm{p}_{1 / 2}$ & $2 \mathrm{p}_{3 / 2}$ & $2 \mathrm{p}_{1 / 2}$ & $2 \mathrm{p}_{3 / 2}$ & $2 \mathrm{p}_{1 / 2}$ \\
\hline nZIF-8 (Zn) & 398.6 & 1021.9 & 1045.0 & & & & & & \\
\hline Atomic ratio & 1.0 & 0.68 & & - & & & - & \\
\hline nZIF-8 (Zn/Ti) & 398.6 & 1021.4 & 1044.5 & & & & & 459.5 & 466.9 \\
\hline Atomic ratio & 1.0 & 0.41 & & - & & & 0.45 & \\
\hline nZIF-67 (Co) & 398.8 & & & 855.2 & 873.0 & 861.4 & 879.2 & & \\
\hline Atomic ratio & 1.0 & - & & 0.58 & & & & - & \\
\hline nZIF-67 (Co/Ti) & 398.9 & & & & & & 459.4 & 465.2 \\
\hline Atomic ratio & 1 & - & & 0.43 & & 0.27 & \\
\hline
\end{tabular}

In addition to XPS, Inductively Coupled Plasma-Optical Emission Spectroscopy (ICP-OES) was used to determine the metallic content ( $\mathrm{Zn}, \mathrm{Co}$, and $\mathrm{Ti}$ ) of the ZIF materials. After digestion of the ZIF crystal materials in an aqueous $\mathrm{HNO}_{3}$ solution, ICP-OES showed a Ti:Zn ratio in nZIF-8(Zn/Ti) and Ti:Co ratio in nZIF-67(Co/Ti) of 1:1 (52\% Ti) and 0.7:1 (39\% Ti) (average of the three measurements) respectively. These values are in good agreement with those obtained by XPS and EDS (Table 2).

Table 2

Ti-content (mol\%) of exchanged ZIFs as measured by XPS, ICP-OES and EDS.

\begin{tabular}{|lll|}
\hline Technique & nZIF-8 (Ti/Zn) mol\% & nZIF-67 (Ti/Co) mol\% \\
\hline XPS & 52 & 38 \\
ICP-OES & 52 & 39 \\
EDS & 51 & 38 \\
\hline
\end{tabular}

All the ZIF materials exhibit type I adsorption isotherms (Fig. 5a), which indicates that they are microporous [26-30]. Both nZIF-8 and nZIF-67 showed a small decrease in the amount of $\mathrm{N}_{2}$ adsorbed, BET surface areas, and pore volumes after metal exchange with Ti(IV) (Table 3). These decreases are in good agreement with the slight decrease in crystallinity, as shown by PXRD measurements (Fig. 2). The TGA profiles in Figure 5(b), show that nZIF-8 and its Ti-exchanged counterpart have the highest thermal stability, with temperatures up to $500^{\circ} \mathrm{C}$, while nZIF-67 and its Ti-exchanged counterpart can withstand temperatures up to $400^{\circ} \mathrm{C}$. After the exchange reaction with Ti(IV), the thermal stability of both nZIF-8 and nZIF-67 decreased only marginally as expected, consistent with literature [7-9]. 
Table 3

Summary of physisorption and Band gap energy data for the nZIF-8, nZIF-67 and their Ti-exchanged derivatives.

\begin{tabular}{|lclllll|}
\hline Sample & \%Yield & $\begin{array}{l}\text { BET } \\
\text { Surface } \\
\text { area/ } \\
\mathbf{m}^{2} \mathbf{g}^{-1}\end{array}$ & $\begin{array}{l}\text { Micropore } \\
\text { Volume/ } \mathbf{c m}^{3} \\
\mathbf{g}^{-1}\end{array}$ & $\begin{array}{l}\text { Average } \\
\text { particle size/ } \\
\mathrm{nm}\end{array}$ & $\begin{array}{l}\text { Decomposition } \\
\text { temperature / } \\
\text { oc }\end{array}$ & $\begin{array}{l}\text { Band gap } \\
\text { Energy / } \\
\text { eV }\end{array}$ \\
\hline $\begin{array}{l}\text { nZIF- } \\
8(\mathrm{Zn})\end{array}$ & 63 & 1867.6 & 0.811 & 48 & 500 & 5.00 \\
\hline $\begin{array}{l}\text { nZIF- } \\
8(\mathrm{Zn} / \mathrm{Ti})\end{array}$ & 92 & 1797.2 & 0.724 & 62 & 450 & 5.01 \\
$\begin{array}{l}\text { nZIF- } \\
67(\mathrm{Co})\end{array}$ & 61 & 1418.5 & 0.548 & 109 & 400 & 2.01 \\
\hline $\begin{array}{l}\text { nZIF- } \\
67(\mathrm{Co} / \mathrm{Ti})\end{array}$ & 88 & 1396.4 & 0.419 & 126 & 350 & 2.00 \\
\hline
\end{tabular}

\section{Figure 5}

The (a) Nitrogen $\left(\mathrm{N}_{2}\right)$ adsorption isotherms $\left(77 \mathrm{~K}\right.$ ) and (b) TGA thermograms (under $\mathrm{N}_{2}$ ) of nZIF-8, nZIF67 , and their Ti-exchanged counterparts.

The band gaps of nZIF-8, nZIF-67 and their Ti-Exchanged derivatives were determined by UV-Vis absorption measurements at room temperature (Figure 6). The band gap of a material is an indication of the minimum energy required for an excited electron to exit the valence band into the conduction band in order to participate in conduction [45]. The ease of excitation may be directly correlated to the effective absorption of visible light, such as artificial sunlight irradiation in this study, by the material and essentially contributing to a more efficient photocatalytic performance [46]. All absorbance spectra have broad absorbance bands in the visible region. The curve of $\left(a^{\star} E\right)^{2}$ versus $E$ (where $a$ is an absorption coefficient and $E$ is the photon energy) [38], was plotted as shown in Fig. 6. The band gap energies of all ZIF materials were determined by the extrapolation of the gradients to the x-axes (where $E=E_{g}$ (the apparent band gap energy)) and the results shown in Table 3. The absorbance peak of pristine nZIF$8(\mathrm{Zn})(300-360 \mathrm{~nm})$ corresponds to a band gap of $5.00 \mathrm{eV}$ (previous reports: $5.2 \mathrm{eV}$ and $4.9 \mathrm{eV}$ ) [38, 47], while the broad absorbance peak of Ti-exchanged nZIF-8(Zn/Ti) $(320-480 \mathrm{~nm})$ corresponds to a band gap of $5.01 \mathrm{eV}$. The very high absorbance around $230 \mathrm{~nm}$ involved $\pi \rightarrow \pi^{\star}$ transitions in the imidazole rings [38]. The absorbance peak of nZIF-67 $(500-650 \mathrm{~nm})$ corresponds to an $\mathrm{E}_{\mathrm{g}}$ of $2.01 \mathrm{eV}$ (reported literature value: $1.98 \mathrm{eV}$ ) [47], with the $E_{g}$ of Ti-exchanged $n Z I F-67(C o / F e)$ at $2.00 \mathrm{eV}$. The $E_{g}$ values indicate that the semiconductor properties of the ZIF-8, ZIF-67 and their respective titanium exchanged derivatives, are similar. The introduction of titanium into the ZIF framework had no appreciable effect on the band gap energies, and therefore no expected effect on the photocatalytic performance of the newly synthesized catalysts. 
Figure 6

The UV-Vis spectra and the Tauc's plots of (a1-a2) nZIF-8(Zn) with nZIF-8(Zn/Ti), (b1-b2) nZIF67(Co) with nZIF-67(Co/Ti).

\section{Photocatalytic Application}

\subsection{Photocatalytic oxidation of Hydroquinone $\left(\mathrm{H}_{2} \mathrm{Q}\right)$ to Benzoquinone (BQ)}

Scheme 3. Photocatalytic oxidation of hydroquinone by $\mathrm{H}_{2} \mathrm{O}_{2}$ and $\mathrm{ZIF}$ catalysts [ZIF-8, ZIF-67, ZIF8(Zn/Ti), ZIF-67(Co/Ti)] Experimental conditions were varied according to i, ii, iii and iv.

The photocatalytic activities of Ti-exchanged nZIF-8(Zn/Ti) and nZIF-67(Co/Ti), and their parent nZIF-8 $(\mathrm{Zn})$ and nZIF-67(Co), were tested in a photocatalytic oxidation transformation of hydroquinone $\left(\mathrm{H}_{2} \mathrm{Q}\right)$ to benzoquinone (BQ), under simulated sunlight irradiation (Scheme 3 ). First, the oxidation effect of the catalyst was evaluated by irradiating $\mathrm{H}_{2} \mathrm{Q}$ (i) alone (HQ), (ii) in the presence of $\mathrm{H}_{2} \mathrm{O}_{2}(\mathrm{HP}$ ), (iii) in the presence of the catalyst $(\mathrm{HC})$ and lastly (iv) in the presence of $\mathrm{H}_{2} \mathrm{O}_{2}$ and the catalyst (HPC). The mixtures were irradiated (simulated sunlight) for a 1 -hour period and the UV spectra were recorded every 3 min as illustrated in Figure 7(a). The same reaction conditions were repeated without irradiation (in the dark) (Supporting Information, Fig. S3). The starting $\mathrm{H}_{2} \mathrm{Q}$ spectra showed a maximum absorbance at $295 \mathrm{~nm}$ (Fig. 7a). Oxidation in the presence of $\mathrm{H}_{2} \mathrm{O}_{2}$ showed a small increase in absorbance at $245 \mathrm{~nm}$ as a result of $\mathrm{BQ}$ formation (HP). Oxidation in the presence of the catalyst $(\mathrm{HC})$ showed a clear absorbance increase at $245 \mathrm{~nm}$ with a corresponding decrease in absorbance at $295 \mathrm{~nm}$. Combination of the catalyst and $\mathrm{H}_{2} \mathrm{O}_{2}$ (HPC) showed the best activity with a much larger increase in the absorbance at $245 \mathrm{~nm}$ and a complete disappearance of the starting material peak at $295 \mathrm{~nm}$. Although less effective, the catalytic effect of this combination was also observed in the dark (Fig. S3) while the other reaction conditions showed no product formation. Following this observation, the catalytic oxidation of $\mathrm{H}_{2} \mathrm{Q}$ was monitored in real time in the presence of $\mathrm{H}_{2} \mathrm{O}_{2}$ and the catalyst, namely, pristine ZIFs, nZIF-8(Zn), nZIF-67(Co) and their titanium exchanged derivatives, nZIF-8(Zn/Ti) and nZIF-67(Co/Ti).

Figure 7: (a) Effect of the oxidation conditions ( $\mathrm{HQ}=\mathrm{H}_{2} \mathrm{Q}$ alone; $\mathrm{HP}=\mathrm{H}_{2} \mathrm{Q}+\mathrm{H}_{2} \mathrm{O}_{2} ; \mathrm{HC}=\mathrm{H}_{2} \mathrm{Q}+\mathrm{nZIF}$ $\left.8(\mathrm{Zn} / \mathrm{Ti})) ; \mathrm{HPC}=\mathrm{H}_{2} \mathrm{Q}+\mathrm{H}_{2} \mathrm{O}_{2}+\mathrm{nZIF}-8(\mathrm{Zn} / \mathrm{Ti})\right)$ on the reaction products after $1 \mathrm{~h}$ of contact time under irradiation (simulated sunlight) and (b) UV-vis absorption spectra for oxidation of hydroquinone catalysed by $\mathrm{nZIF-8}(\mathrm{Zn} / \mathrm{Ti})$ in the presence of hydrogen peroxide.

Fig. 7(b) shows the overlay UV spectra of nZIF-8(Zn/Ti) catalytic performance, which was similar to that of nZIF-67(Co/Ti) (Supporting Information, Fig. S5). A rapid, exponential decrease in absorbance at 295 $\mathrm{nm}$ and simultaneous increase at $245 \mathrm{~nm}$, corresponds to the disappearance of $\mathrm{H}_{2} \mathrm{Q}$ and the formation of $\mathrm{BQ}$ respectively. The oxidative fraction $\left(\mathrm{A} / \mathrm{A}_{\circ}\right)$ decreased progressively and reached $100 \%$ for both nZIF- 
$8(\mathrm{Zn} / \mathrm{Ti})$ and $\mathrm{nZIF}-67(\mathrm{Co} / \mathrm{Ti})$ after $36 \mathrm{~min}$. A first-order catalytic behaviour was confirmed by the linear plots of $\ln (A / A \infty)$ versus time (Fig. 8). The individual $k_{B Q}$ rate constants for the formation of $B Q$ were calculated as $0.189 \mathrm{~min}^{-1}\left(\mathrm{t}_{1 / 2}=3.7 \mathrm{~min}\right)$ and $0.139 \mathrm{~min}^{-1}\left(\mathrm{t}_{1 / 2}=5 \mathrm{~min}\right)$ for nZIF-8(Zn/Ti) and nZIF$67(\mathrm{Co} / \mathrm{Ti}$ ) respectively (Table 4$)$. Comparison of the influence of the metal exchange on the rate of oxidation showed that the Ti-exchanged nZIF-8( $\mathrm{Zn} / \mathrm{Ti})$ and nZIF-67(Co/Ti) were approximately 4.4 and 4.8 times faster than the parent nZIF-8 and nZIF-67 respectively (Fig. 9). It is evident that the incorporation of $\mathrm{Ti}(\mathrm{IV})$-centres into the ZIF framework improved the rate of oxidation dramatically when compared to the pristine ZIFs, (Supporting Information, Fig. S4).

The introduction of a second metallic component may improve catalytic performance due to the expected synergistic effect between the two metals. This effect is caused by variations in the valence electronic configuration, difference in surface composition and the presence of different metal oxidation states $\left[{ }^{48,49}\right]$. The increase in oxidation rate for the bimetallic derivatives can be attributed to an increase in the number of active sites on the surface of the photocatalyst with more $\mathrm{Ti}^{4+}$ exposed, which increased the electron transfer rate to the oxidant to drive the process of photo-oxidation of hydroquinone [45]. This is evident with the high oxidation rate on Ti-exchanged ZIFs. A higher titanium content ( $52 \mathrm{~mol} \%$ ) of the nZIF-8(Zn/Ti) catalyst which represents a Ti:Zn ratio of nearly $1: 1$, compared to that of nZIF-67(Co/Ti) (38 $\mathrm{mol} \%$ ), led to higher photocatalytic activity. It is certain that incorporation of $\mathrm{Ti}^{4+}$ into the $\mathrm{ZIF}$ framework, improved their photocatalytic activity dramatically (Fig. 9). The influence of the particle size and external surface area on the rate of activity was also analysed. Smaller particles ( $48 \mathrm{~nm})$ of the nZIF-8(Zn/Ti) catalyst gave 1.4 times faster oxidation rate, compared to the bigger particles $(\sim 110 \mathrm{~nm})$ of nZIF-67(Co/Ti), due to a larger surface area. The conversion of $\mathrm{H}_{2} \mathrm{Q}$ to $\mathrm{BQ}$ was confirmed by FTIR

(Fig. 10) with a disappearance of a broad $\mathrm{OH}$ stretching, sharp $\mathrm{OH}$ bonding and $\mathrm{C}-\mathrm{O}$ stretching frequency of the $\mathrm{H}_{2} \mathrm{Q}$ at $3200 \mathrm{~cm}^{-1}$ (i), $1480 \mathrm{~cm}^{-1}$ (iii) and $1200 \mathrm{~cm}^{-1}$ (iv) respectively, as well as the appearance of a sharp $\mathrm{C}=0$ stretching frequency at $1700 \mathrm{~cm}^{-1}$ (ii), associated with the formation of $B Q$.

\section{Figure 8}

$A / A_{0}$ curves for decomposition of Hydroquinone reagent and formation of Benzoquinone product in the

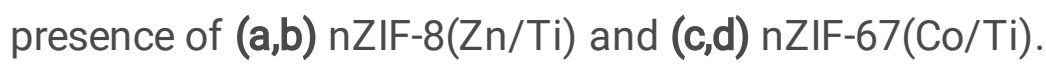


Table 4

$\mathrm{K}_{\mathrm{obs}}$ and $\mathrm{t}_{1 / 2}$ for nZIF-8(Zn), nZIF-67(Co) and their Ti-exchanged derivatives on photocatalytic oxidation of hydroquinone.

\begin{tabular}{|lllll|}
\hline Sample & $\mathbf{k}_{\mathrm{H} 2 \mathrm{Q}}\left(\mathrm{min}^{-1}\right)$ & $\mathrm{t}_{1 / 2}(\min )_{\mathrm{H} 2 \mathrm{Q}}$ & $\mathrm{k}_{\mathrm{BQ}}\left(\mathrm{min}^{-1}\right)$ & $\mathrm{t}_{1 / 2}(\mathrm{~min})_{\mathrm{BQ}}$ \\
\hline $\mathrm{nZIF-8}(\mathrm{Zn})$ & $4.49 \times 10^{-2}$ & 15.4 & $4.29 \times 10^{-2}$ & 16.2 \\
\hline nZIF-67(Co) & $2.09 \times 10^{-2}$ & 33.2 & $2.89 \times 10^{-2}$ & 23.9 \\
\hline Exchanged nZIF-8(Zn/Ti) & $17.9 \times 10^{-2}$ & 3.9 & $18.95 \times 10^{-2}$ & 3.7 \\
\hline Exchanged nZIF-67(Co/Ti) & $14.7 \times 10^{-2}$ & 4.7 & $13.9 \times 10^{-2}$ & 5.0 \\
\hline
\end{tabular}

Figure 9

Comparison of rate constants for the production of Benzoquinone (BQ) during the photocatalytic oxidation of hydroquinone $\left(\mathrm{H}_{2} \mathrm{Q}\right)$ in the presence of pristine and Ti-exchanged ZIF catalysts.

\section{Figure 10}

FTIR curves of hydroquinone reagent and benzoquinone product in the presence of Ti-exchanged ZIF catalysts.

\subsection{Stability and reusability test after 3 cycles of photocatalysis}

The Ti-exchanged ZIFs were taken through two additional catalytic cycles of oxidation. After every cycle, the Ti/ZIF-8 and Ti/ZIF-67 catalysts were separated from the reaction medium by centrifugation and washed with $\mathrm{MeOH}$ multiple times until supernatant was colourless. The catalyst was thereafter dried at $60^{\circ} \mathrm{C}$ and activated at $150^{\circ} \mathrm{C}$ under vacuum. The photocatalytic activity of the regenerated Ti-exchanged ZIF towards oxidation of $\mathrm{H}_{2} \mathrm{Q}$ was almost equal to that of the fresh catalyst. A slight reduction in activity (less than 10\%) was observed after three subsequent cycles (Supporting Information, Fig. S11), indicating the catalyst good stability for oxidation of $\mathrm{H}_{2} \mathrm{Q}$. The slight decrease in the catalytic activity of the recycled catalyst may be ascribed to the loss of product during regeneration and the surface area as a result of the ZIF particle tendency to aggregate, thereby reducing the number of available catalytic sites. This tendency is common amongst porous catalyst materials. Although the angle reflection intensity was reduced, the XRD patterns of the recycled Ti/ZIF-8 and Ti/ZIF-67 catalysts were found to be identical to the fresh catalysts as evidenced by the retention of the angles of reflection in Figure 11. This confirms that the sodalite framework of the Ti-exchanged ZIFs was preserved, and no visible structural changes occurred after catalysis.

Figure 11: (a) The FTIR spectra and (b) PXRD patterns of nZIF-8(Zn/Ti) and nZIF-67(Co/Ti) catalysts after three photocatalytic cycles of oxidation of hydroquinone. 


\section{Conclusion}

In conclusion, the Zn and Co nodes of nano-sized ZIF-8(Zn) and ZIF-67(Co) were partially replaced by $\mathrm{Ti}(\mathrm{IV})$. The Ti centre was introduced by postsynthetic metal-exchange to give the first bimetallic metal exchanged microporous Ti(IV)-containing ZIFs, nZIF-8(Zn/Ti) and nZIF-67(Co/Ti). The morphology, crystal structures and the structural integrity of the Sodalite framework of the pristine ZIF nanoparticles have been retained to a large extend during the Ti-exchange process. Their BET surface areas and pore volume decreased by less than $5 \%$ and binding energy values on XPS confirmed the preservation of the oxidation state of $\mathrm{Zn}(\mathrm{II}), \mathrm{Co}(\mathrm{II})$ and Ti(IV) after the exchange process. The catalytic activity of the pristine nZIF-8(Zn) and nZIF-67(Co) was enhanced by approximately 4.5 times for the photocatalytic oxidation of $\mathrm{H}_{2} \mathrm{Q}$ to benzoquinone by incorporating Titanium ions in their structures. The presence of the Ti(IV)centres in the ZIF structures proved to be a good promoter for the performance of the two catalysts. The band gap energy of the ZIF materials had essentially no effect on the photocatalytic performance and was predominated by the nature of the active site and surface area. Easy recovery of the Ti-exchanged ZIFs was achieved by decantation and the catalyst was reusable up to three times without any substantial loss in the catalytic activity and crystal stability.

\section{Declarations}

\section{Statements \& Declarations}

\section{Conflicts of Interest}

The authors declare that they have no conflicts of interest.

\section{Acknowledgements}

The authors gladly acknowledge the financial assistance provided by the SASOL grant from the Department of Chemistry at the University of the Free State. We also thank Professor E. Erasmus for XPS analysis, the Analytical Chemistry Department for their assistance with the ICP-OES study, Ithemba Labs (Cape Town) for the PXRD analysis, and the Centre for Microscopy (UFS) for the SEM-EDX analysis.

\section{References}

[1] H. Zhou, J.R. Long, and O.M. Yaghi, Chem. Rev. (2012) https://doi.org/10.1021/cr300014x.

[2] S. Biswas and N. Stock, Chem. Rev. (2012) https://doi.org/10.1021/cr200304e.

[3] S.L. Griffin and N.R. Champness, Coord. Chem. Rev. (2020) https://doi.org/10.1016/j.ccr.2020.213295

[4] D. Banerjee and J.B Parise, Cryst. Growth Des. (2011) https://doi.org/10.1021/cg2008304.

[5] A. Zanon,F. Verpoort, Chem. Rev. (2017) https://doi.org/10.1016/j.ccr.2017.09.030. 
[6] Y. Liu, H. Cheng, M. Cheng, Z. Liu, D. Huang, G. Zhang, B. Shao, Q. Liang, S. Luo, T. Wu and S. Xiao, Chem. Eng. J. (2021) https://doi.org/10.1016/j.cej.2020.127914.

[7] K.K. Tanabea and S.M. Cohen, Chem. Soc. Rev. (2011) https://doi.org/10.1039/C0CS00031K.

[8] L.E. Mphuthi, E. Erasmus, E.H.G. Langner, ACS Omega. (2021)

https://doi.org/10.1021/acsomega.1c04142.

[9] M. Kim, J. Cahill, H. Fei, K. Prather and S. Cohen, J. Am. Chem. Soc. (2012)

https://doi.org/10.1021/ja3079219.

[10] H. Assi, G. Mouchaham, N. Steunou, T. Devic and C. Serre, Chem. Soc. Rev. (2017)

https://doi.org/10.1039/C7CS00001D

[11] M. A. Nasalevich , M. van der Veen , F. Kapteijn and J. Gascon , Cryst. Eng. Comm. (2014) https://doi.org/10.1039/C4CE00032C

[12] S. Abedia and A. Morsali , New J. Chem. (2015) https://doi.org/10.1039/C4NJ01536C

[13] H.L. Nguyen, New J. Chem. (2017) https://doi.org/10.1039/C7NJ03153J

[14] J. Zhu, P. Li, W. Guo, Y. Zhao and R. Zou, (2018) https://doi.org/10.1016/j.ccr.2017.12.013

[15] H.L. Nguyen, J. Phys. Energy. (2021) https://doi.org/10.1088/2515-7655/abe3c9

[16] Y. Yan, C. Li, Y. Wu, J. Gao and Q. Zhang, J. Mater. Chem. A, (2020) https://doi.org/10.1039/D0TA03749D

[17] W.H. Fang, L. Zhang and J. Zhang, Chem. Soc. Rev. (2018) https://doi.org/10.1039/C7CS00511C

[18] J. Zhu, P.Z. Li, W. Guo, Y. Zhao and R. Zou, Coord. Chem. Rev. (2018)

https://doi.org/10.1016/j.ccr.2017.12.013

[19] C. Tsai, J.W. Niemantsverdriet and E.H.G. Langner, Microporous Mesoporous Mater. (2018) https://doi.org/10.1016/j.micromeso.2017.11.024

[20] X. Fan, W. Wang, W. Li, J. Zhou, B. Wang, J. Zheng and X. Li, ACS Appl. Mater. Interfaces. (2014) https://doi.org/10.1021/am5028346

[21] Y. Li, K. Zhou, M. He and J. Yao, Microporous Mesoporous Mater. (2016) https://doi.org/10.1016/j.micromeso.2016.07.039

[22] H. Fei, J. Cahill, K. Prather and S. Cohen, Inorg. Chem. (2013) https://doi.org/10.1021/ic400048g

[23] E. Guibal, T. Vincent, E. Touraud, S. Colombo, A. Ferguson, J. Appl. Polym. Scie. 4, 100 (2006). 
[24] F. Moulder, W.F. Stickle, P.E. Sobol, K.D. Bomben, Handbook of X-ray Photoelectron Spectroscopy, ULVAC-PHI, Inc., Enzo, Chigasaki, Japan, 1995.

[25] M.R.A. Hamid, S. Park, J.S. Kim, Y. M. Lee and H. Jeong, J. Mater. Chem. A. (2019) https://doi.org/10.1039/C9TA00837C

[26] M. Jian, B. Liu, R. Liu, J. Qu, H. Wang and X. Zhang, RSC Adv. (2015) https://doi.org/10.1039/C5RA04033G

[27] S.R. Venna, J.B. Jasinski and M.A. Carreon, J. Am. Chem. Soc. (2010) https://doi.org/10.1021/ja109268m

[28] S. Tanaka, K. Kida, M. Okita, Y. Ito, Y. Miyake, Chem. Lett. (2012) https://doi.org/10.1246/cl.2012.1337

[29] J. Zaręba, M. Nyk and M. Samoć, Cryst. Growth Des. (2016)

https://doi.org/10.1021/acs.cgd.6b01090

[30] W. Sun, X. Zhai and L. Zhao, Chem. Eng. J. 289 (2016) http://dx.doi.org/10.1016/j.cej.2015.12.076

[31] M. Kim, J. Cahill, H. Fei, K. Prather and S. Cohen, J. Am. Chem. Soc. (2012) https://doi.org/10.1021/ja3079219

[32] I.U. Khan, M.H.D. Othman, A. Jilani, A.F Ismail, H. Hashim, J. Jaafar, M.A Rahman and G.U. Rehman, Arabian J. Chem. (2018) https://doi.org/10.1016/j.arabjc.2018.07.012

[33] X. Fan, W. Wang, W. Li, J. Zhou, B. Wang, J. Zheng and X. Li, ACS Appl. Mater. Interfaces. (2014) https://doi.org/10.1021/am5028346

[34] Y. Li, K. Zhou, M. He and J. Yao, Microporous Mesoporous Mater. (2016) https://doi.org/10.1016/j.micromeso.2016.07.039

[35] F. Song, Y. Cao, Y. Zhao, R. Jiang, Q. Xu, J. Yan and Q. Zhong, J. Nanomater. (2020) https://doi.org/10.1155/2020/1508574

[36] G. Greczynski, L. Hultman, Chem. Phys. Chem. (2017) https://doi.org/10.1002/cphc.201700126.

[37] T.R. Gengenbach, G.H. Major, M.R. Linford, C.D. Easton, J. Vacuum Scie. Tech. A. (2021) https://doi: 10.1116/6.0000682.

[38] M. Thanh, T. Thien, P. Du, N. Hung, D. Khieu, J. Por. Mater. (2018) https://doi.org/10.1007/s10934017-0498-7

[39] W. Wu, J. Su, M. Jia, Z. Li, G. Liu and W. Li, Sci. Adv. (2020) https://doi.org/10.1126/sciadv.aax7270 
[40] J.M. Lindquist, J.P. Ziegler, J.C. Hemminger, Surface Scie. (1989) https://doi.org/10.1016/00396028(89)90101-5

[41] A. Nqombolo, T.S. Munonde, T.A. Makhetha, R.M. Moutloali, P.N. Nomngongo, J. Mater. Res. Tech. (2021) https://doi.org/10.1016/j.jmrt.2021.03.113

[42] J.C. Carver, G.K. Schweitzer, T.A. Carlson, J. Chem. Phys. (1972). https://doi.org/10.1063/1.1678348.

[43] M.C. Biesinger, L.W.M. Lau, A. Gerson and R.St.C. Smart. Appl. Surf. Scie. (2010) https://doi.org/10.1016/j.apsusc.2010.07.086

[44] U. Diebold, Surface Science Spectra. (1996) https://doi.org/10.1116/1.1247794.

[45] Y. Xu, M.A.A. Schoonen, American Mineralogist. (2000) https://doi.org/10.2138/am-2000-0416.

[46] T. Bak, W. Li, J. Nowotny, A.J. Atanacio and J. Davisc, J. Phys. Chem. A, (2015) https://doi.org/10.1021/acs.jpca.5b05031.

[47] D. Saliba, M. Ammar, M. Rammal, M. Al-Ghoul and M. Hmadeh, J. Am. Chem. Soc. (2018) https://doi.org/10.1021/jacs.7b11589

[48] A. Alshammari, V.N. Kalevaru and A. Martin, Catalysts. (2016) https://doi.org/10.3390/catal6070097 [49] G.A. Somorjai, Y. Li, Introduction to Surface Chemistry and Catalysis; John Wiley \& Sons: Hoboken, NJ, USA, 2010.

\section{Scheme}

Please see the Supplementary Files for the Scheme 1, 2 and 3.

\section{Figures}

\section{Figure 1}

FTIR spectra of (a) nZIF-8(Zn) with nZIF-8(Zn/Ti) and (b) nZIF-67(Co) with nZIF-67(Co/Ti). 

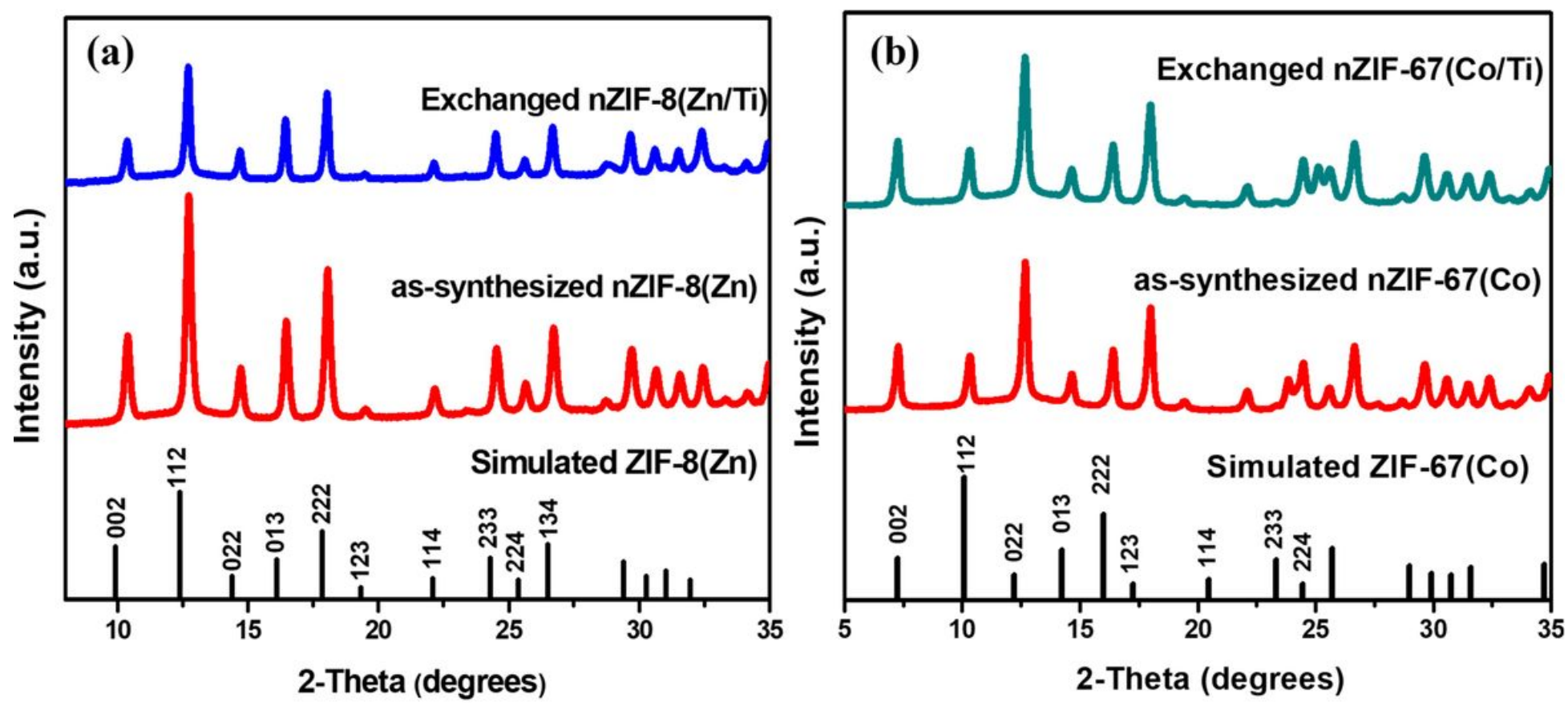

Figure 2

PXRD patterns of (a) nZIF-8(Zn) with nZIF-8(Zn/Ti) and (b) nZIF-67(Co) with nZIF-67(Co/Ti).

Figure 3

SEM images of (a) ZIF-8(Zn), (b) nZIF-8(Zn/Ti), (d) nZIF-67(Co), (e) nZIF-67(Co/Ti) and EDX-mapping of Ti-exchanged nZIF-8(Zn/Ti) (C1-C4) and nZIF-67(Co/Ti) (F1-F4).

Figure 4

The (a) N 1s, (b) Zn 2p, (c) Ti 2p and (d) Co 2p XPS regions of ZIF-8, ZIF-67 and their Ti-exchanged derivatives. 

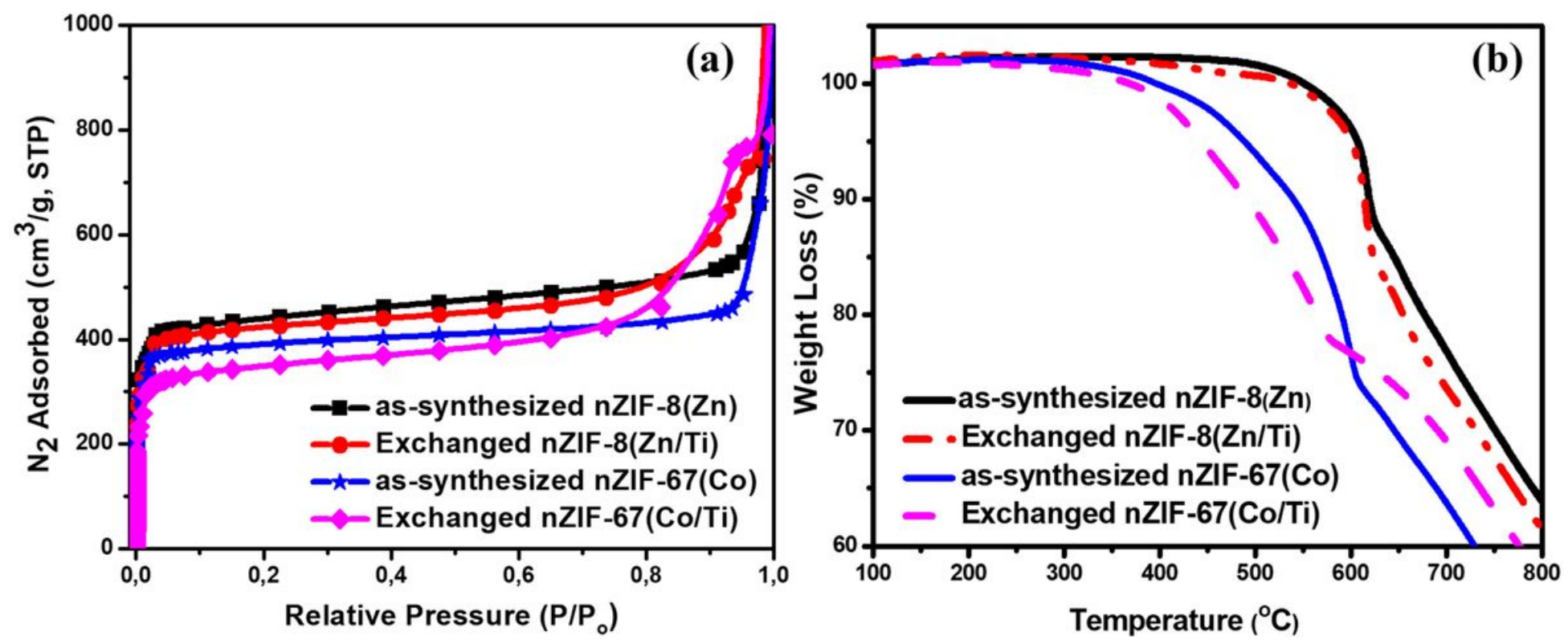

Figure 5

The (a) Nitrogen $\left(\mathrm{N}_{2}\right)$ adsorption isotherms (77 K) and (b) TGA thermograms (under $\mathrm{N}_{2}$ ) of nZIF-8, nZIF67 , and their Ti-exchanged counterparts. 

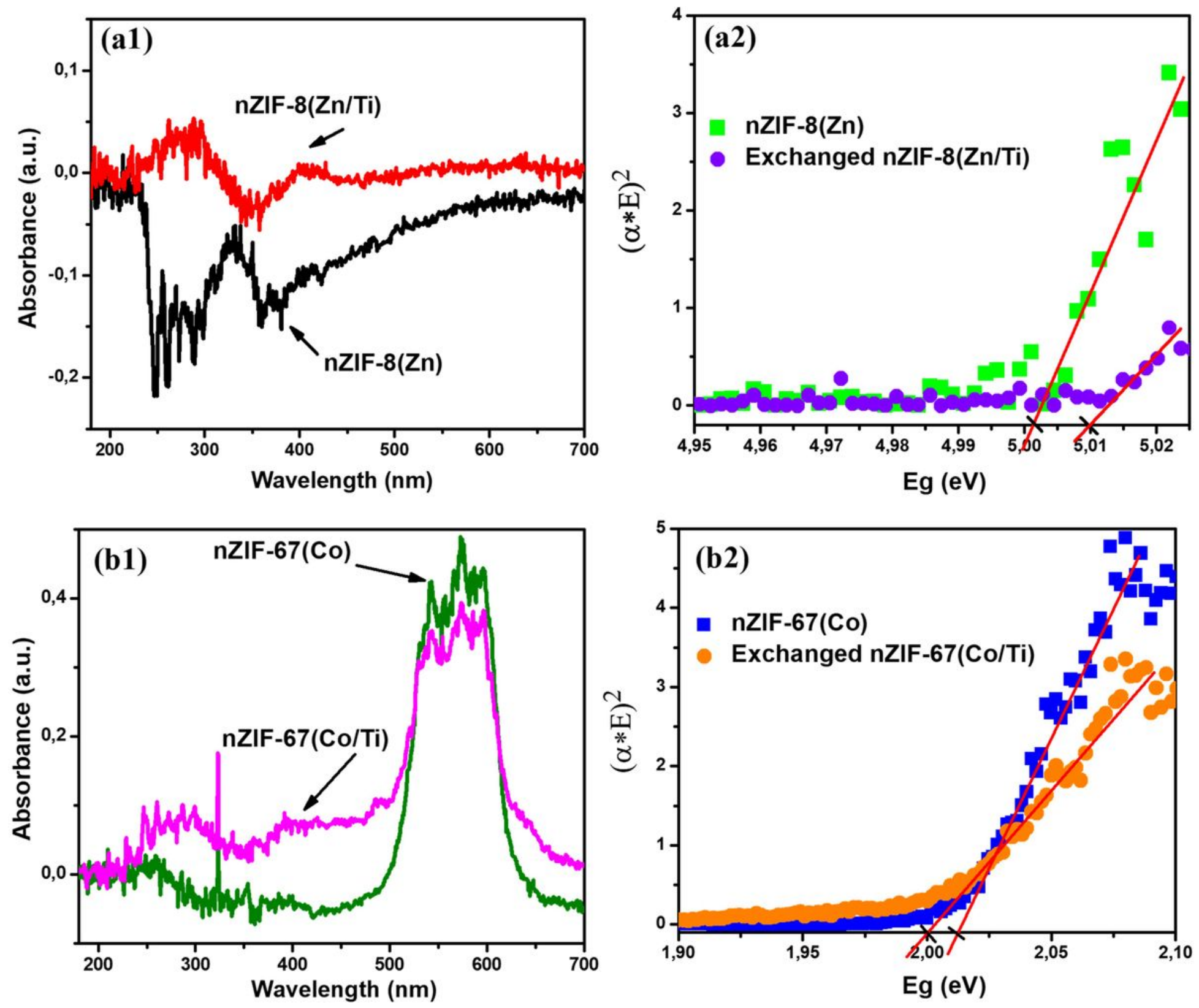

Figure 6

The UV-Vis spectra and the Tauc's plots of (a1-a2) nZIF-8(Zn) with nZIF-8(Zn/Ti), (b1-b2) nZIF67(Co) with nZIF-67(Co/Ti).

Figure 7

(a) Effect of the oxidation conditions ( $\mathrm{HQ}=\mathrm{H}_{2} \mathrm{Q}$ alone; $\left.\mathrm{HP}=\mathrm{H}_{2} \mathrm{Q}+\mathrm{H}_{2} \mathrm{O}_{2} ; \mathrm{HC}=\mathrm{H}_{2} \mathrm{Q}+\mathrm{nZIF}-8(\mathrm{Zn} / \mathrm{Ti})\right) ; \mathrm{HPC}$ $\left.=\mathrm{H}_{2} \mathrm{Q}+\mathrm{H}_{2} \mathrm{O}_{2}+\mathrm{nZIF-8}(\mathrm{Zn} / \mathrm{Ti})\right)$ on the reaction products after $1 \mathrm{~h}$ of contact time under irradiation (simulated sunlight) and (b) UV-vis absorption spectra for oxidation of hydroquinone catalysed by nZIF$8(\mathrm{Zn} / \mathrm{Ti})$ in the presence of hydrogen peroxide. 
(a)
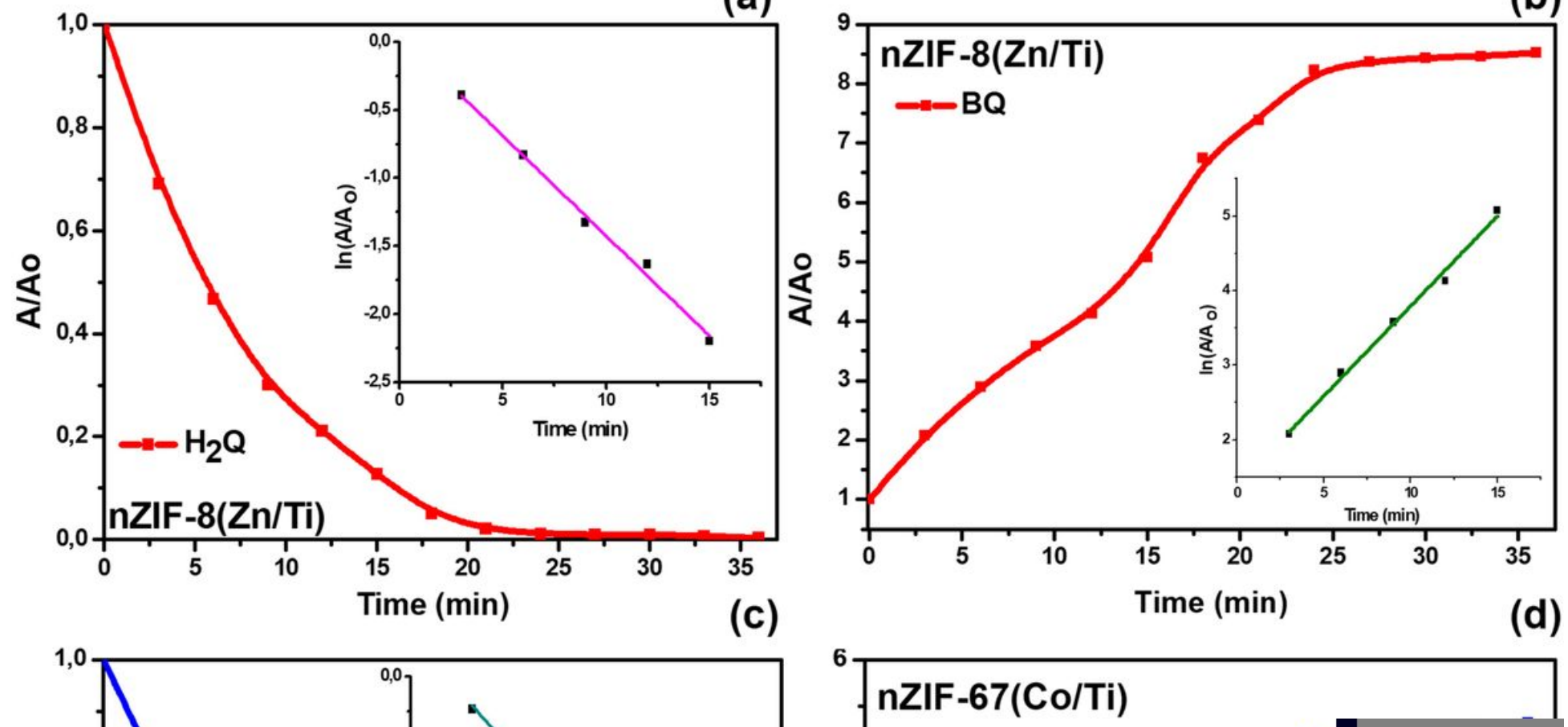

Figure 8

$\mathrm{A} / \mathrm{A}_{0}$ curves for decomposition of Hydroquinone reagent and formation of Benzoquinone product in the presence of $(\mathbf{a}, \mathbf{b})$ nZIF-8(Zn/Ti) and (c,d) nZIF-67(Co/Ti). 


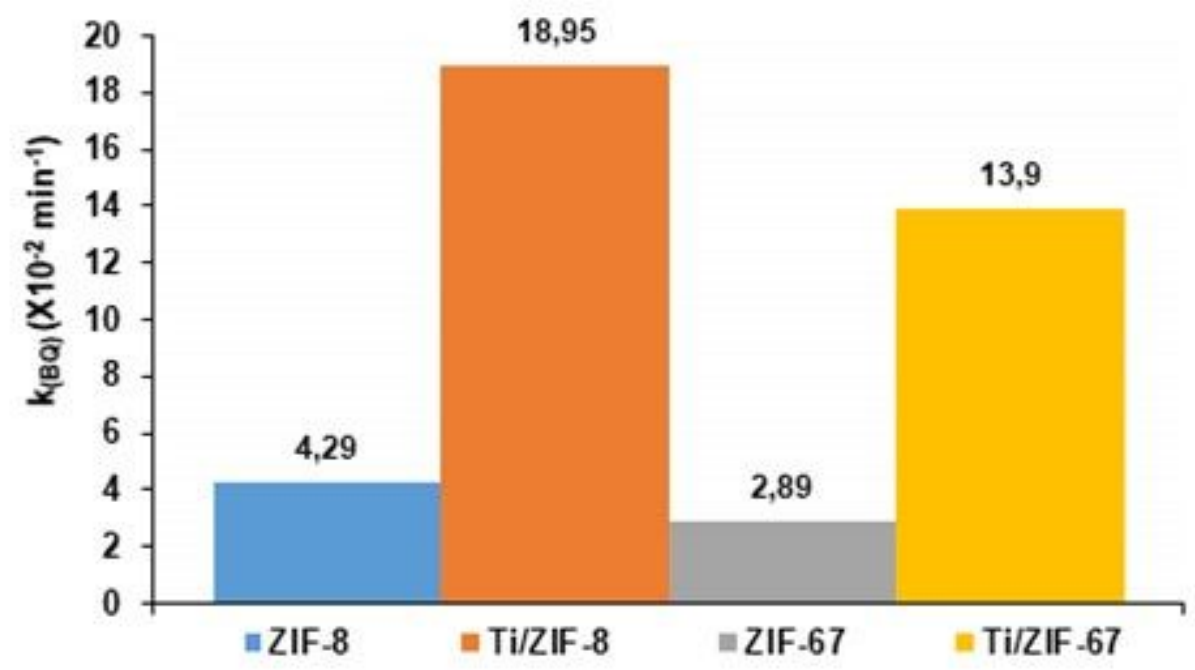

Figure 9

Comparison of rate constants for the production of Benzoquinone (BQ) during the photocatalytic oxidation of hydroquinone $\left(\mathrm{H}_{2} \mathrm{Q}\right)$ in the presence of pristine and Ti-exchanged ZIF catalysts. 


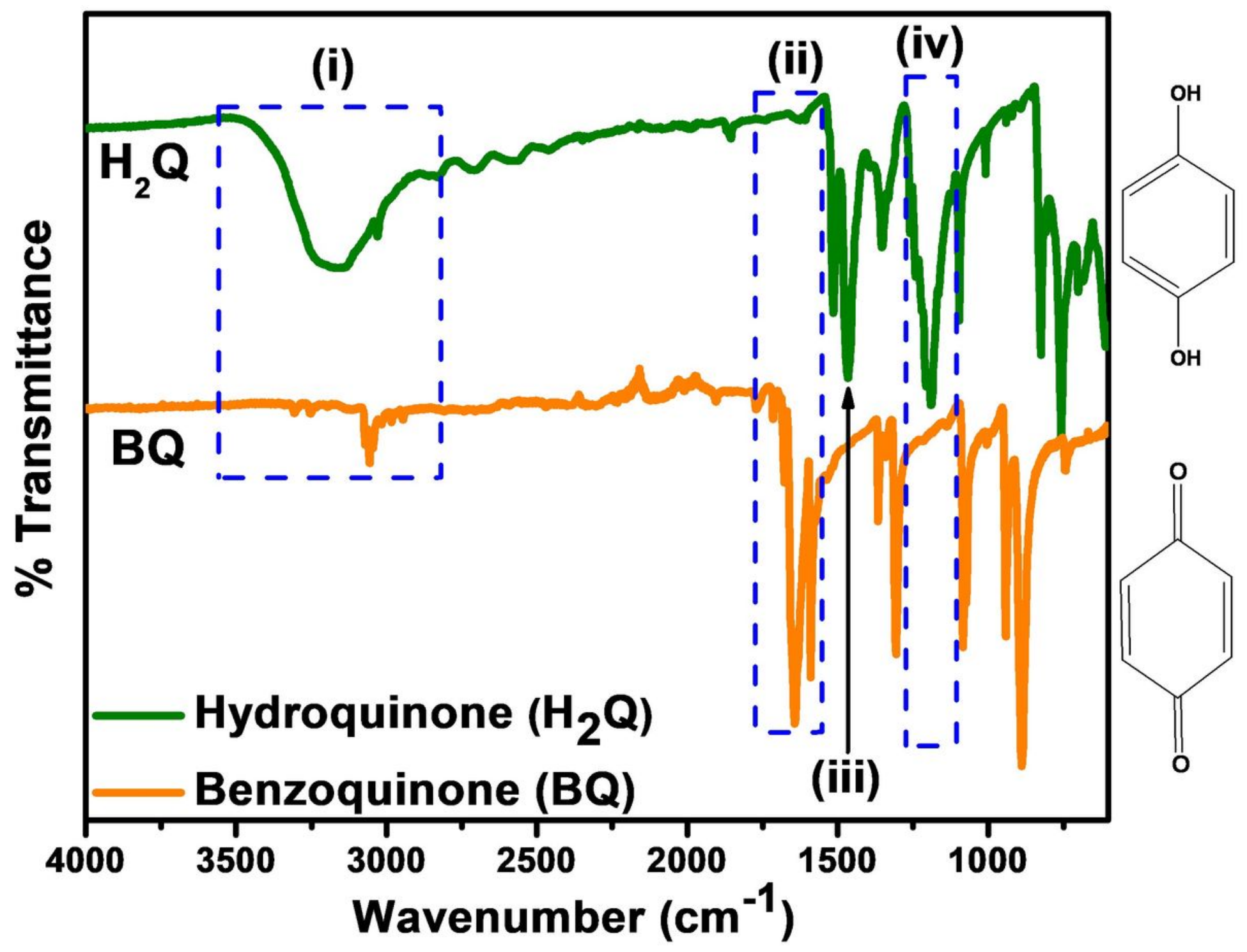

Figure 10

FTIR curves of hydroquinone reagent and benzoquinone product in the presence of Ti-exchanged ZIF catalysts. 
(a)

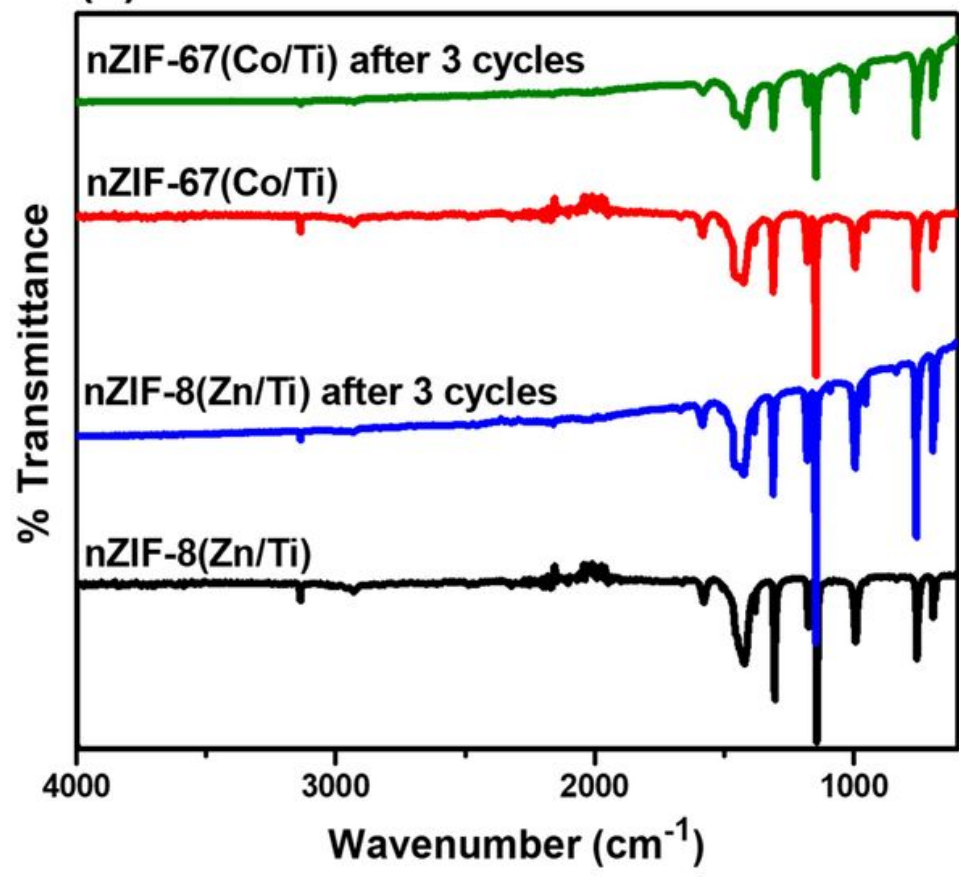

(b)

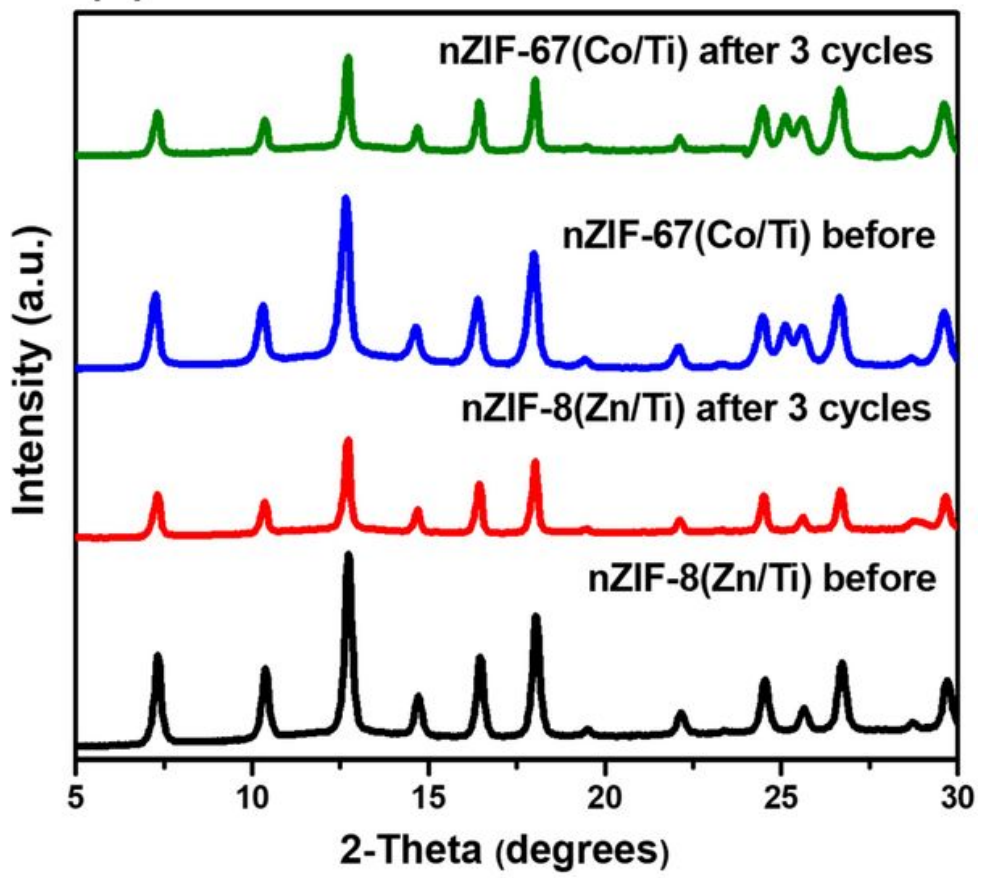

Figure 11

This confirms that the sodalite framework of the Ti-exchanged ZIFs was preserved, and no visible structural changes occurred after catalysis.

(a) The FTIR spectra and (b) PXRD patterns of nZIF-8(Zn/Ti) and nZIF-67(Co/Ti) catalysts after three photocatalytic cycles of oxidation of hydroquinone.

\section{Supplementary Files}

This is a list of supplementary files associated with this preprint. Click to download.

- Graphicalabstract.jpg

- LEMphuthiTiZIFsJIOPMSuppInfo.pdf

- Scheme1.jpg

- Scheme2.jpg

- Scheme3.jpg 